\title{
THE RELATIONSHIP BETWEEN DIRECT DETERMINATION AND FD-GRAPH
}

\author{
HO THUAN, NGUYEN VAN DINH
}

\begin{abstract}
The notion of direct determination was introduced by D. Maier [5] to study the structure of minimum covers. Using direct determination he showed that it is possible to find covers with the smallest number of FDs (Functional Dependencies) in polynomial time. In [2], G. Ausiello et al. presented an approach which is based on the representation of the set of FDs by FD-graph (considered as a special case of the hypergraph formalism introduced in [7]). Such a representation provides a unified framework for the treatment of various properties and for the manipulation of FDs.
\end{abstract}

In this paper, we establish the relation between FD-graph and direct determination, and prove some well-known and new properties concerning direct determination.

Tóm tăt. Khái niệm xác định trực tiếp đã được trình bày bơ‘i D. Maier [5] đễ nghiên cưu cấu trúc các phư cực tiểu. Sử dụng khái niệm này, ông đã chl ra rằng có thể tìm được các phư với số phụ thuộc hàm là ít nhất trong thòi gian đa thức. Trong [2], G. Ausiello và các tác giả khác đã đưa ra một cách tiếp cận mới trên cơ sở biễu diễn tập các phụ thuộc hàm bằng một FD-đồ thị (xem như một trường hộp đặc biệt của siêu đồ thị, được giới thiệu trong [7]). Cách biểu diễn như vậy cho một khung thống nhất để xử lý nhiều tính chất khác nhau và thao tác trên các FD.

Trong bài báo này, chúng tôi xác định mối liên hệ giữa FD-đồ thị và khái niệm xác định trực tiếp, chứng minh một số tính chất quen biết và những tính chất mới liên quan đến khái niệm này.

\section{BASIC NOTIONS AND RESULTS}

In this section we recall some notions and results which will be needed in the sequel. The reader is required to know the basic notions of the relational model and functional dependency [8]. As usual, we will only consider sets of FD in natural reduced form [4] and we assume that all attributes are chosen from some fixed universe $\Omega$. That means for any $F=\left\{X_{i} \rightarrow Y_{i} \mid i=1,2, \ldots, m\right\}$

$$
\begin{aligned}
& X_{i} \cap Y_{i}=\emptyset, \forall i=1,2, \ldots, m ; \\
& X_{i} \neq X_{j} \text { for } i \neq j ; \\
& X_{i}, Y_{i} \subseteq \Omega, \forall i=1,2, \ldots, m .
\end{aligned}
$$

Let $F^{+}$be the closure of $F$, i.e. the set of all FDs that can be inferred from the FDs in $F$ by repeated application of the Armstrong's axioms [1].

\section{Definition 1.1.}

(a) Two sets $F_{1}, F_{2}$ of FDs over $\Omega$ are said equivalent, written $F_{1} \equiv F_{2}$ if $F_{1}^{+}=F_{2}^{+}$. If $F_{1} \equiv F_{2}$ then $F_{1}$ is a cover for $F_{2}$ and vice versa.

(b) A set $F$ of FDs is nonredundant if there is no proper subset $F^{\prime}$ of $F$ with $F^{\prime} \equiv F$. $F_{1}$ is a nonredundant cover for $F_{2}$ if $F_{1}$ is a cover for $F_{2}$ and $F_{1}$ is nonredundant.

(c) Let $F$ be a set of FDs over $\Omega$ and let $X \rightarrow Y$ be a FD in $F$. Attribute $A \in \Omega$ is said extraneous in $X \rightarrow Y$ if

$$
((F \backslash\{X \rightarrow Y\}) \cup\{X \backslash A \rightarrow Y \backslash A\})^{+}=F^{+} .
$$

(d) Two set of attributes $X$ and $Y$ are equivalent under a set of FDs, written $X \leftrightarrow Y$, if $X \rightarrow Y$ and $Y \rightarrow X$ are in $F^{+}$. 
Definition 1.2. [5] Given a set of FDs $F$ with $X \rightarrow Y$ in $F^{+}$. $X$ direct determines $Y$ under $F$,

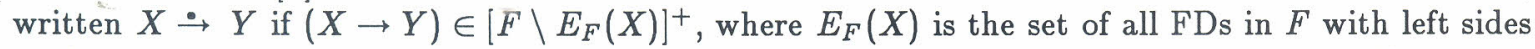
equivalent to $X$. That is, no FDs with left sides equivalent to $X$ are used to derive $X \rightarrow Y$.

Definition 1.3. [5] A set of FDs F is minimum if there is no set $G$ with fewer FD than $F$ such that $G \equiv F$.

Theorem 1.1. [5] Given equivalent minimum set of FDs $F$ and $G$

$$
\left|E_{F}(X)\right|=\left|E_{G}(X)\right| \text { for any } X \text {. }
$$

Thus the size of equivalence classes in $\bar{E}_{F}$ is the same for all minimum $F$ with the same closure (where $\bar{E}_{F}$ is the collection of all non empty $E_{F}(X)$ ).

Definition 1.4. [2] Given a set of FDs on $\Omega$, the FD-graph $G_{F}=\langle V, E\rangle$ associated with $F$ is the graph with node labeling function $w: V \rightarrow P(\Omega)$ and are labeling function $w^{\prime}: E \rightarrow\{0,1\}$ such that:

(i) for every attribute $A \in \Omega$, there is a node in $V$ labeled $A$ (called simple node);

(ii) for every dependency $X \rightarrow Y$ in $F$ where $|X|>1$, there is a node in $V$ labeled $X$ (called a compound node);

(iii) for every dependency $X \rightarrow Y$ in $F$ where $Y=A_{1} \ldots A_{k}$, there are arcs labeled 0 (full arcs) from the node labeled $X$ to the nodes labeled $A_{1}, \ldots, A_{k}$;

(iv) for every compound node $i$ in $V$ labeled $A_{i_{1}} \ldots A_{i_{p}}$ there are arcs labeled 1 (dotted arcs) from the node $i$ to all simple nodes (component nodes of $i$ ) labeled $A_{i_{1}}, \ldots, A_{i_{p}}$.

The set of full arcs (dotted arcs, respectively) is denoted $E_{0}\left(E_{1}\right.$, respectively).

Example 1.1. Given a set of attributes $\Omega=\{A, B, C, D, E, F, H\}$, let $F$ be a set of FDs over $\Omega$, $F=\{A \rightarrow B C F, C \rightarrow D, F B D \rightarrow H, B D \rightarrow E\}$ the corresponding FD-graph $G_{F}=\langle V, E\rangle$ is shown in Fig. 1.1.

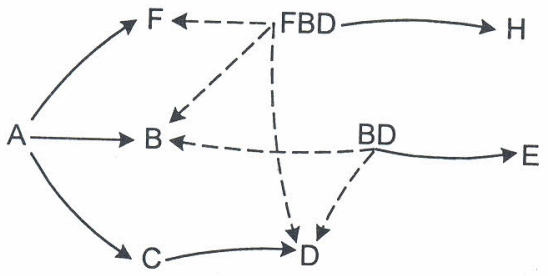

Fig.1.1. An FD-graph

Definition 1.5. [2] Given an FD-graph $G_{F}=\langle V, E\rangle$ and two nodes $i, j \in V$, a (directed) FD-path $\langle i, j\rangle$ from $i$ to $j$ is a minimal subgraph $\bar{G}_{F}=\langle\bar{V}, \bar{E}\rangle$ of $G_{F}$ such that $i, j \in \bar{V}$ and either $(i, j) \in \bar{E}$ or one of the following possibilities holds:

(a) $j$ is a simple node and there exists a node $k$ such that $(k, j) \in \bar{E}$ and there is an FD-path $\langle i, k\rangle$ included in $\bar{G}_{F}$ (graph transitivity).

(b) $j$ is a compound node with component nodes $m_{1}, \ldots, m_{r}$ and there dotted $\operatorname{arcs}\left(j, m_{1}\right), \ldots,\left(j, m_{r}\right)$ in $\bar{G}_{F}$ and $r$ FD-paths $\left\langle i, m_{1}\right\rangle, \ldots,\left\langle i, m_{r}\right\rangle$ included in $\bar{G}_{F}$ (graph union).

Further more, an FD-path $\langle i, j\rangle$ is dotted if all its arcs leaving $i$ are dotted; otherwise it is full.

Example 1.2. For the FD-graph of the Example 1.1: (a) full FD-path $\langle A, E\rangle$, (b) full FD-path $\langle A, D\rangle$, and dotted FD-path $\langle F B D, E\rangle$ are given in Fig. 1.2. 
(a)

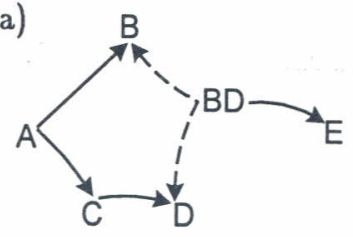

(b)

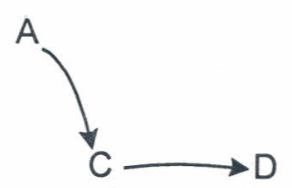

(c)

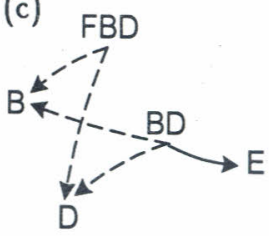

Fig. 1.2. FD-paths

Definition 1.6. [2]

(a) The closure of an FD-graph $G_{F}=\langle V, E\rangle$ is the graph $G_{F}{ }^{+}=\left\langle V, E^{+}\right\rangle$, labeled on the nodes and on the arcs, where the set $V$ is the same as in $G_{F}$, while the set $E^{+}=\left(E^{+}\right)_{0} \cup\left(E^{+}\right)_{1}$ is defined in the following way

$\left(E^{+}\right)_{1}=\{(i, j) \mid i, j \in V$ and there exists a dotted FD-path $\langle i, j\rangle\}$ $\left(E^{+}\right)_{0}=\left\{(i, j) \mid i, j \in V,\left(i, j \notin\left(E^{+}\right)_{1}\right.\right.$ and there exists a full FD-path $\left.\langle i, j\rangle\right\}$.

(b) Two nodes $i, j$ in an FD-graph are said equivalent if the arcs $(i, j)$ and $(j, i)$ both belong to the closure of $G_{F}$. Further more, a node $i$ of $G_{F}$ is said to be equivalent to node $j$ of $G_{\bar{F}}$ where $G_{\bar{F}}$ is a cover of $G_{F}$ (i.e. $F^{+}=\bar{F}^{+}$) if $i, j$ are equivalent in some cover of $G_{F}$.

(c) Given two FD-graphs $G_{F_{1}}, G_{F_{2}} ; G_{F_{2}}$ is a cover of $G_{F_{1}}$ if $F_{2}$ is a cover of $F_{1}$.

(d) An FD-graph $G_{F}$ is nonredundant if $F$ is nonredundant.

Theorem 1.2. [2] Let $G_{F}=\langle V, E\rangle$ be the FD-graph associated with the set $F$ of $F D$, and let $G_{F}{ }^{+}=\left\langle V, E^{+}\right\rangle$be its closure. An arc $(i, j)$ is in $E^{+}$if and only if $w(i) \rightarrow w(j)$ is in $F^{+}$.

Theorem 1.3. [2] $A$ nonredundant $F D$-graph $G_{F}=\langle V, E\rangle$ is minimum if and only if it has no superfluous node.

Recall that a node $i \in V$ is superfluous if there exists a dotted FD-path $\langle i, j\rangle$ where $j$ is a node of $V$ equivalent to $i$.

\section{DIRECT DETERMINATION AND FD-GRAPH}

In this section, we establish the relation between FD-graph and direct determination by proving some well-known and new properties of direct determination.

First it is worth giving a few comments on the definition of an FD-graph.

Remark 2.1. Definition 1.4 is reasonable and concise in the sense that the FD-graph $G_{F}$ includes all the "meaning part" of the closure of the set of FDs. On the other hand, with the formalism of FD-graph, we can provide a simple and unified treatment of all properties of sets of FDs.

Following the definition of a FD-graph, it is clear that every compound node has at least one outgoing full arc. However, according to the necessity, we can freely add to an FD-graph some new coumpound nodes without outgoing full arcs if it makes easy to prove a certain required property.

So, a natural way is to think that an FD-graph $G_{F}=\langle V, E\rangle$ associated with $F$ is defined by Definition 1.4 precisely to an arbitrary finite number of different compound nodes which do not correspond to the left side of any FD in $F$, together with the dotted arcs from each of them to their corresponding component nodes.

Definition 2.1. [2] Given an FD-graph $G_{F}=\langle V, E\rangle$ and a node $i \in V$ with at least a full outgoing arc. A strong component of $G_{F}$ with representative node $i$ is a maximal set of pairwise equivalent nodes which contains $i$, denoted by $\mathrm{SC}(i)$. Notice that every node in $\mathrm{SC}(i)$ has at least one full outgoing arc.

The following lemma is obvious. 
Lemma 2.1. Given an $F D$-graph $G_{F}=\langle V, E\rangle$, a node $i \in V$, its corresponding strong component $S C(i)$ and two nodes $j, k$ such that $j$ is equivalent to $i$. ( $j$ not necessarily belong to $S C(i)$, i.e. $j$ can be a compound node without outgoing full arc that we add it to the FD-graph. The same situation can happen with the node $k$ too).

Then $w(j) \stackrel{\rightarrow}{\rightarrow} w(k)$ if and only if there exists a dotted $F D$-path $\langle j, k\rangle$ containing no full outgoing arc from any node of $S C(i)$.

In other words, the dotted FD-path $\langle j, k\rangle$ contains no intermediate node that is node of $S C(i)$.

In that case, for sake of simplicity, we write $\langle j \stackrel{\mathrm{SC}(i)}{\longrightarrow} k\rangle$.

Example 2.1. Given $\Omega=A B C D E I H, F=\{A \rightarrow B C H, B C \rightarrow A, A D \rightarrow E I, E A \rightarrow I D\}$. It is easy to verify that:

$$
E_{F}(A D)=\{A D \rightarrow E I, A E \rightarrow D I\} \text { and } B C D \leftrightarrow A D .
$$

The corresponding FD-graph $G_{F}$ with an added node $B C D$ (without outgoing full arc) is shown in Fig. 2.1.

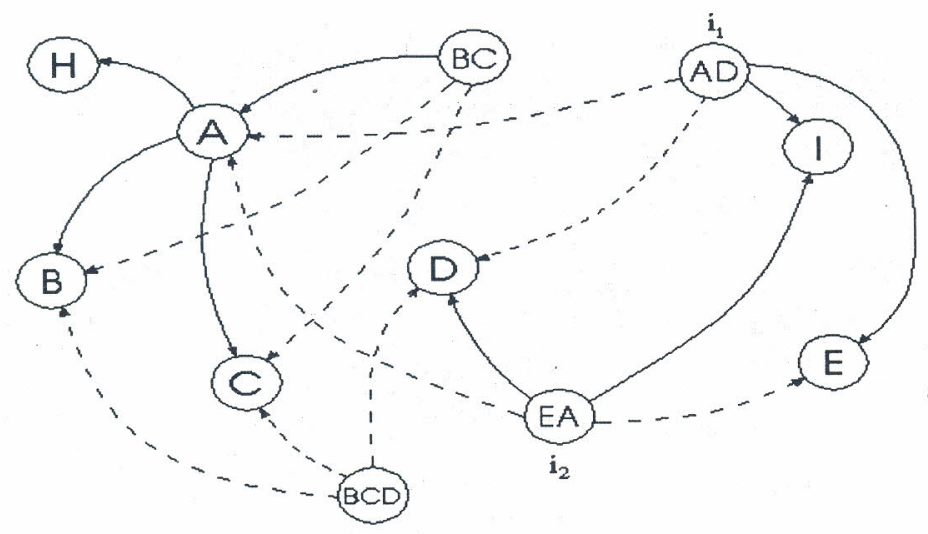

Fig. 2.1. FD-graph with added node $B C D$

We have

we find that

$$
\operatorname{SC}\left(i_{1}\right)=\left\{i_{1}, i_{2}\right\} \text { where } w\left(i_{1}\right)=A D, w\left(i_{2}\right)=E A,
$$

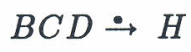

and

$$
B C D \doteq A D \text {. }
$$

Lemma 2.2. Given an $F D$-graph $G_{F}=\langle V, E\rangle$, two equivalent nodes $i, j \in V$ and $i_{q}, j_{q}$ are two nodes equivalent to $i$ and $j$ respectively.

$$
\text { If }\left\langle i_{q} \stackrel{\mathrm{SC}(i)}{\longrightarrow} j_{q}\right\rangle \text { and }\left\langle j_{q} \stackrel{\mathrm{SC}(j)}{\longrightarrow} k\right\rangle \text { then }\left\langle i_{q} \stackrel{\mathrm{SC}(i)}{\longrightarrow} k\right\rangle \text {. }
$$

Proof. By merging two FD-paths $\left\langle i_{q} \stackrel{\mathrm{SC}(i)}{\longrightarrow} j_{q}\right\rangle$ and $\left\langle j_{q} \stackrel{\mathrm{SC}(j)}{\longrightarrow} k\right\rangle$ appropriately at compound nodes of $j_{q}$ which are intermediate nodes of the FD-path $\left\langle j_{q} \stackrel{\mathrm{SC}(j)}{\longrightarrow} k\right\rangle$ we obtain the FD-path $\left\langle i_{q} \stackrel{\operatorname{SC}(i)}{\longrightarrow} k\right\rangle$.

In other words, from $w(i) \leftrightarrow w\left(i_{q}\right), w(j) \leftrightarrow w\left(j_{q}\right)$ and $w\left(i_{q}\right) \bullet w\left(j_{q}\right), w\left(j_{q}\right) \leftrightarrow w(k)$, we have $w\left(i_{q}\right) \dot{\rightarrow} w(k)$.

Notice that the above lemma corresponds to [5, Lemma 5].

Example 2.2. Take up again Example 2.1 (Fig. 2.1), we have $B C D \rightarrow A D$ and $A D \rightarrow H$.

Since $A$ is the unique component node of $A D$ that is an intermediate node on the FD-path 
$\left\langle A D \stackrel{\mathrm{SC}\left(i_{1}\right)}{\longrightarrow} H\right\rangle$, we will merge two FD-paths $\langle B C D, A D\rangle$ and $\langle A D, H\rangle$ at $A$ to obtain the FD-path $\langle B C D, H\rangle$ such that $B C D \stackrel{\rightarrow}{H}$.

Lemma 2.3. Given an $F D$-graph $G_{F}=\langle V, E\rangle, i \in V$ is a node having at least one outgoing full arc and $i_{0}$ is equivalent to $i$ ( $i_{0}$ can be an added node to the FD-graph without outgoing full arc). Then there exists $j \in S C(i)$ such that $\left\langle i_{0} \stackrel{\mathrm{SC}(i)}{\longrightarrow} j\right\rangle$.

Proof. Suppose that $i_{0} \notin \mathrm{SC}(i)$. Otherwise, take $j \equiv i_{0}$ and the lemma is proved. Consider the dotted FD-path $\left\langle i_{0}, i\right\rangle$. In the case there is no intermediate node in $\left\langle i_{0}, i\right\rangle$ that is node of $\operatorname{SC}(i)$ then $i$ is the node to be found.

Otherwise, suppose that $i_{1} \in \mathrm{SC}(i)$ is an intermediate node of $\left\langle i_{0}, i\right\rangle$. Now we have only to consider the FD-path $\left\langle i_{0}, i_{1}\right\rangle$. Repeat the above reasoning for $\left\langle i_{0}, i_{1}\right\rangle$. Finally, we will find the required $j$ such that $\left\langle i_{0} \stackrel{\operatorname{SC}(i)}{\longrightarrow} j\right\rangle$.

Notice that the above lemma corresponds to [5, Lemma 6].

Lemma 2.4. Let $G_{F}=\langle V, E\rangle$, be a minimum $F D$-graph (i.e. $F$ is minimum), and $i \in V$ is a node with at least one outgoing full arc. Then in $S C(i)$ there exist no $j_{1}, j_{2} ; j_{1} \neq j_{2}$ such that $\left\langle j_{1} \stackrel{\operatorname{SC}(i)}{\longrightarrow} j_{2}\right\rangle$.

Proof. Assume the contrary that there exist $j_{1}, j_{2} \in \mathrm{SC}(i), j_{1} \neq j_{2}$ such that there is a dotted FDpath from $j_{1}$ to $j_{2}$. Since $j_{1}$ is equivalent to $j_{2}, j_{1}$ is a superfluous node. We arrive to a contradiction. (See Theorem 1.3).

Notice that the above lemma corresponds to [5, Lemma 7].

Lemma 2.5. Given two nonredundant $F D$-graph $G_{F_{1}}=\left\langle V_{1}, E_{1}\right\rangle, G_{F_{2}}=\left\langle V_{2}, E_{2}\right\rangle$, wherein $G_{F_{2}}$ is a cover of $G_{F_{1}}$. Let $i_{1}$ and $i_{2}$ be two equivalent nodes in $V_{1}$ and $V_{2}$, respectively, with at least one outgoing full arc, $\left(p_{2}, q_{2}\right)$ be a full arc of $E_{2}$ with $p_{2} \neq S C^{(2)}\left(i_{2}\right) .{ }^{(*)}$ If $\left(i_{1}, p_{2}\right) \in E_{2}{ }^{+}$, then $\left\langle p_{2} \stackrel{\mathrm{SC}^{(1)}\left(i_{1}\right)}{\longrightarrow} q_{2}\right\rangle$.

Proof. Since $\left(i_{1}, p_{2}\right) \in E_{2}{ }^{+}$, by Theorem 1.2, there exists a FD-path in $G_{F_{1}}$ from $i_{1}$ to $p_{2}$. Now assume the contrary that the FD-path in $G_{F_{1}}$ from $p_{2}$ to $q_{2}$ has an intermediate node $j_{1} \in \mathrm{SC}^{(1)}\left(i_{1}\right)$. The presence of the FD-path $\left\langle j_{1}, i_{1}\right\rangle$ shows that $p_{2}$ is equivalent to $i_{1}$, i.e. $p_{2} \in \operatorname{SC}^{(2)}\left(i_{2}\right)$, a contradition.

Theorem 2.6. With the same assumptions as in Lemma 2.5, if we replace in $G_{F_{1}}$ all nodes belonging to $S C^{(1)}\left(i_{1}\right)$ together with their corresponding outgoing arcs by all nodes in $S C^{(2)}\left(i_{2}\right)$ together with their corresponding outgoing arcs, then the new FD-graph is a cover of $G_{F_{1}}$.

Proof. We have only to prove that for every full arc $\left(j_{1}, k_{1}\right) \in E_{1}$ with $j_{1} \in \operatorname{SC}^{(1)}\left(i_{1}\right)$ there exists a FD-path $\left\langle j_{1}, k_{1}\right\rangle$ in the new FD-graph. By Lemma 2.5 we have just the required result.

Remark 2.2. Theorem 2.6 can be formulated in another form as follows:

If $F_{1}, F_{2}$ are nonredundant and equivalent sets of $\mathrm{FDs}$, then

$$
F_{1} \equiv\left\{F_{1} \backslash E_{F_{1}}(X)\right\} \cup E_{F_{2}}(X) \equiv\left\{F_{2} \backslash E_{F_{2}}(X)\right\} \cup E_{F_{1}}(X) .
$$

Let us close the paper with the following useful proposition:

Proposition 2.7. Let $U \rightarrow W$ be an FD in $F^{+}$and let $X \rightarrow Y$ be an $F D$ in $F$ that participates in the Armstrong's derivation sequence for $U \rightarrow W$. Then we have:

$$
U \rightarrow X, U Y \rightarrow W \in(F \backslash\{X \rightarrow Y\})^{+} .
$$

$\mathrm{SC}^{(1)}$ and $\mathrm{SC}^{(2)}$ refer to $G_{F_{1}}$ and $G_{F_{2}}$, respectively 
Proof. Let $G_{F}=\langle V, E\rangle$ be the FD-graph associated with $F$. From $U \rightarrow W$ in $F^{+}$it follows that there is an FD-path $\langle i, j\rangle$ from $i$ to $j$, where $w(i)=U, w(j)=W$. Since $X \rightarrow Y \in F$ takes part in the derivation sequence for $U \rightarrow W$, the nodes $p$ and $q$ with $w(p)=X$ and $w(q)=Y$ are intermediate nodes on $\langle i, j\rangle$. It is clear that the FD-paths $\langle i, p\rangle$ and $\langle q, j\rangle$ contain no outgoing full arc from node $p$.

Example 2.3. Reconsider the Example 2.1 (Fig. 2.1). We have $B C D \rightarrow H \in F^{+},(B C \rightarrow A) \in F$ participates in the derivation sequence for $B C D \rightarrow H$.

It is clear that:

$B C D \rightarrow B C \in(F \backslash\{B C \rightarrow A\})^{+}$and corresponds to the FD-path $\langle B C D, B C\rangle$;

$B C D A \rightarrow H \in(F \backslash\{B C \rightarrow A\})^{+}$and corresponds to the FD-path $\langle B C D A, H\rangle$.

\section{CONCLUSIONS}

An FD-graph approach for the representation of functional dependencies (FDs) in relational databases. It also supports the studies of FDs. This approach allow a homogeneous treatment of several problems (closure, minimization, etc.), which leads to simpler proofs and, in some cases, more efficient algorithms than in the current literature. Therefore, the studies of FD-graph is a middle step to further study Database Hypergraphs in which directed hyperedges represent FDs and undirected hyperedges represent the join dependency.

\section{REFERENCES}

[1] Armstrong W. W., Dependency structures of database relationships, Information Processing 74, North Holland Publishing Company, 1974, 580-583.

[2] Ausiello G. et al., Graphs algorithms for functional dependency manipulation, J. ACM 30 (1983) 752-766.

[3] Fagin R., Ling Ling Yan, Renee J. Miller, and Laura M. Haas, Data-driven understanding and refinement of schema mappings, Proc. 2001 ACM SIGMOD Symposium, Santa Barbara, 485-496.

[4] Ho Thuan, Contribution to the Theory of Relational Database, Tanulmányok, 184/1986, Budapest, Hungary.

[5] Maier D., Minimum covers in the relational database model, J. ACM 27 (1980) 664-674.

[6] S. Nguyen, D. Pretolani, and L. Markenzon, Some path problems on oriented hypergraphs, Theoretical Informatics and Applications (Elsevier-Paris) 32 (1998), No. 1, 2, 3.

[7] Saccá D., Closures of database hypergraphs, J. ACM 32 (1985) 774-803.

[8] Ullman Jeffrey D., Principles of Database and Knowledge-Base Systems, Computer Science Press, USA, 1989.

Received October 25, 2001

Ho Thuan, National Institute of Information Technology, Hanoi.

Nguyen Van Dinh, United Nations International School of Hanoi. 